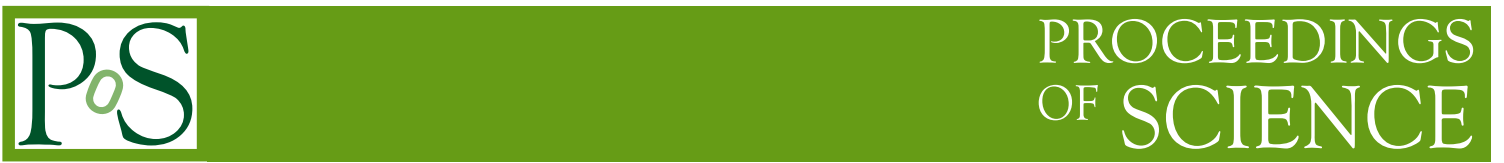

\title{
Top quark properties from CDF and Do
}

\author{
Sung Keun Park* \\ On behalf of the CDF and DO collaboration \\ Korea Detector Laboratory, Korea University \\ Seoul, 136-701, Korea \\ E-mail: sungpark@korea.ac.kr
}

Recent results on top quark properties measured by the CDF and the D0 collaborations are presented, including the top quark charge using a $b$-jet charge algorithm and soft lepton tagger, spin-correlations in the helicity basis and in the beam line basis, the $\mathrm{W}$ boson helicity in top quark decay, the forward-backward charge asymmetry.

XVIII International Workshop on Deep-Inelastic Scattering and Related Subjects April 19 -23, 2010

Convitto della Calza, Firenze, Italy

${ }^{*}$ Speaker.

${ }^{\dagger}$ I acknowledge the support of Fermilab through its International Fellowship Program, and Korea University. 


\section{Introduction}

The discovery of the top quark at the Tevatron completed the standard model with three generations of quarks [2,3]. The apparent oddity of the top quark is its mass: about 40 times larger than the mass of its companion $b$-quark in their weak-isospin doublet. In its decay to a $\mathrm{W}$ boson and a $b$-quark, it has a short lifetime, on a time scale of order $\frac{1}{\Gamma_{t}} \approx 5 \times 10^{-25} \mathrm{~s}$. The subsequent hadronization of the $b$-quark takes place on a time scale of $\frac{1}{\Lambda_{Q C D}}$. If this is an accurate description of the evolution of the top quark, then the top quark remains "bare" without forming any of its possible bound states during its lifetime. This provides a unique window to study the top quark as a "bare quark". And it makes the study of the top quark properties more interesting. For example, the spin information carried by the top quark is transferred to its decay products without the interference of spin-spin interactions from its hardronization $[4,5]$.

The study of top quark properties requires of a good understanding of the production and decay mechanisms of the top quark. The top quark at a hadron collider is predominantly produced via the strong interaction, and the production cross section of $t \bar{t}$ is a convolution of the parton distribution functions $(p d f)$ in the colliding hadrons.

At the Tevatron energy of $1.96 \mathrm{TeV}$, the momentum fraction of a parton in the proton is about 0.18 where the $p d f$ of the $u$-quark is larger than that of the gluon, while the $p d f$ of the $d$-quark is comparable to that of the gluon. At this energy top quarks are produced predominantly as $t \bar{t}$ pairs. So the main processes of $t \bar{t}$ production are the $q \bar{q} \rightarrow t \bar{t}$ and $g g \rightarrow t \bar{t}$ processes. The $q \bar{q} \rightarrow t \bar{t}$ process comprises $85 \%$ of the production cross section, and the $g g \rightarrow t \bar{t}$ process comprises the remaining $15 \%$ [6].

Due to the different $t \bar{t}$ production dynamics, the top quark spin effects can be exploited both at the Tevatron and the LHC. For example, since $q \bar{q}$ annihilation dominates $t \bar{t}$ production at the Tevatron, while $g g$ annihilation dominates at the LHC, the spin correlation is expected to have the opposite sign at these colliders [7].

The top quark decays via the weak interaction, and assuming the CKM matrix element $V_{t b}$ to be close to 1 , the top quark decays always into a $\mathrm{W}$ boson and a $b$-quark. A $t \bar{t}$ event will have two $b$-quarks and two $\mathrm{W}$ bosons. Directly from the decay modes of the $\mathrm{W}$ boson, the decay channels of the $t \bar{t}$ pair can be classified as follows: the lepton + jets channels where one $\mathrm{W}$ boson decays leptonically and the other decays hadronically, and di-lepton channels where both $\mathrm{W}$ bosons decay leptonically into $e^{+} e^{-}, \mu^{+} \mu^{-}, e \mu$, and the all-hadronic channel where both $\mathrm{W}$ bosons decay hadronically.

\section{Measurements of Top Quark Properties}

\subsection{Top Quark Charge}

The top quark discovered at the Tevatron is expected to be the top quark that was sought after within the SM, and the companion of the $b$-quark in the weak-isospin doublet. Then its electric charge is also expected to be $+\frac{2}{3} e$. But experimentally when $t \bar{t}$ is reconstructed from $W^{+} W^{-} b \bar{b}$, there are two different possibilities in matching the $b$-quark and $\mathrm{W}$ bosons. In the SM combining $\left(W^{+} b\right), t \rightarrow W^{+} b$, will form the expected $(t, b)_{L}$ but the other combination $\left(W^{-} b\right)$ can provide a possibility that an exotic quark $\mathrm{Q}$ with charge $-\frac{4}{3} e$ decays via $Q \rightarrow W^{-} b$. This interesting 
possibility is consistent with current precision electroweak data when a top quark mass of $m_{t}=270$ $\mathrm{GeV}$ is assumed and a right-handed $b$-quark is allowed to mix with an exotic quark $Q_{1}$ of charge $-\frac{1}{3} e$ in the doublet $\left(Q_{1}, Q_{4}\right)_{R}$, where $Q_{4}$ of charge $-\frac{4}{3} e$ is the exotic top quark with higher mass of $270 \mathrm{GeV}$ [8]. In this scenario $Q_{4} \rightarrow W^{-} b, Q_{4}$ is the particle produced and escaped from detection at the Tevatron. Therefore the top quark charge measurements are required to either confirm or rule out one of the hypotheses. The discrimination of the top quark charge between the $\frac{2}{3} e$ and $\frac{4}{3} e$ charge scenarios are carried out by the CDF and the D0 collaborations $[9,10]$.

\subsubsection{DO Result}

The D0 experiment has performed the first experimental discrimination between charge $\frac{2}{3} e$ top quark and charge $\frac{4}{3} e$ exotic quark in the lepton + jets channel with $370 p b^{-1}$ of data. In each selected $t \bar{t}$ event the assignment of the correct decay products of the top quark to the correct top quark was done by a constrained kinematic fit. The observable top quark charge $q_{t o p}$ is obtained as the sum of the lepton charge from the $\mathrm{W}$ boson decaying leptonically and the charge of the associated $b$-jet, $q_{t o p}=\left|q_{l}+q_{b}\right|$. The $b$-jet charge is determined by a $p_{T}$ weighted sum of the charges of the tracks in the $b$-jet. The result of this analysis concludes that the data are consistent with the expected electric charge, $\left|q_{\text {top }}\right|=\frac{2}{3} e$ and excludes at the $92 \%$ C.L that the sample is solely due to the production of exotic quark pairs with $\left|q_{t o p}\right|=\frac{4}{3} e$.

\subsubsection{CDF Result}

The CDF experiment recently reports a measurement of the electric charge of the top quark in the lepton + jets channel using data of $2.7 \mathrm{fb}^{-1}$. The analysis of $t \bar{t}$ event uses a kinematic fitter to determine which $b$-jet is associated with the leptonically or hadronically decaying $\mathrm{W}$ boson. The soft lepton taggers (soft electron tagger and soft muon tagger) and a secondary vertex tagger are used to identify the $b$-jets. Once the $b$-jets are tagged, the soft lepton tagger is used once again to determine the $b$-jet flavor. Each event is classified SM if the product of the charge of lepton decaying from $\mathrm{W}$ boson and the charge of $b$-jet associated with the $\mathrm{W}$ boson is negative. The event is classified XM if it is otherwise. Based on the total number of SM and XM pairs, a normalized asymmetry A is calculated as the test statistic. And the p-value is calculated: XM p-value is 0.0094 . Therefore XM hypothesis is excluded at $95 \%$ confidence level.

\subsection{Spin Correlation}

The top quarks are expected to be produced unpolarized, but their spins are expected to be correlated. Since top quarks decay before their hadronization, their spins at production are transferred to their decay particles. Their spin correlation is analyzed by using the joint decay angular distribution of one decay particle from $t$ and one from $\bar{t}$. Since the down-type fermions (charged leptons or $d$-type quarks) in the top quark decay tends to go in the direction of the top quark spin, good sensitivity to the top quark spin is obtained when the decay particles from top quark are down-type fermions. The double differential distribution for a measurement of spin correlations of $t \bar{t}$ can be as follows:

$$
\frac{1}{\sigma} \frac{d^{2} \sigma}{d \cos \theta_{1} d \cos \theta_{2}}=\frac{1}{4}\left(1-C \cos \theta_{1} \cos \theta_{2}\right)
$$


where $\theta_{1}$ and $\theta_{2}$ are the angle of the decay particles in the top quark rest frames with respect to a spin basis. $C$ is a free parameter between -1 and 1 depending on the choice of the spin basis. In the beam direction spin basis at the Tevatron the maximum value is $C=0.777$ [11]. D0 measured $C$ in the dilepton sample using the neutrino weighting method as used in the top quark mass measurements in the dilepton channel [12]. Using data of $4.2 \mathrm{fb}^{-1}$ they measured $C=-0.17_{-0.53}^{+0.64}$ [13].

Recent CDF analysis used a binned likelihood template fit. Four different Monte Carlo samples were created according to the four possible $t \bar{t}$ helicity states: $t_{R} \bar{t}_{L}, t_{L} \bar{t}_{R}, t_{L} \bar{t}_{L}, t_{R} \bar{t}_{R}$. The opposite helicity template was created by combining the $t_{R} \bar{t}_{L}, t_{L} \bar{t}_{R}$ samples in equal ratios accounting for parity conservation. The same helicity template was created by combining the $t_{L} \overline{t_{L}}, t_{R} \overline{t_{R}}$ in equal ratios accounting for $\mathrm{CP}$ conservation. Using these templates, a binned likelihood fit to data from $4.3 \mathrm{fb}^{-1}$ was performed. The opposite helicity fraction $f_{o}$ was measured: $f_{o}=0.80 \pm$ 0.25 (stat.) \pm 0.08 (syst.). The spin correlation coefficient $\kappa$ was obtained from $f_{o}=\frac{1}{2}(\kappa+1): \kappa=$ $0.60 \pm 0.50$ (stat.) \pm 0.16 (syst.) [14].

\subsection{W Boson Helicity in Top Decay}

When the top quark decays into a $\mathrm{W}$ boson and a $b$-quark, the helicity state of the massive spin-1 $\mathrm{W}$ boson can be in one of three polarization states: longitudinal polarization, left-handed polarization, or right-handed polarization. Since the coupling of W bosons to fermions is V-A in the SM, the top decays to either left-handed $\mathrm{W}$ bosons or longitudinal $\mathrm{W}$ bosons. And the presence of right-handed $\mathrm{W}$ bosons will indicate the non-standard couplings such as V+A. Measurement of the helicity of the $\mathrm{W}$ boson tests the standard model stringently. The prediction of the SM is as follows: the fraction of left-handed $f_{-}=0.7$, the fraction of longitudinal $f_{0}=0.3$, and the fraction of right-handed $f_{+}=0.0$. The angular distribution of the down-type decay products of the $\mathrm{W}$ boson (charged lepton or $d, s$ quark) in the rest frame of the $\mathrm{W}$ boson can be described by introducing the decay angle $\theta^{*}$ of the down-type fermion with respect to the top quark direction. The dependence of the distribution of $\cos \theta^{*}$ on the $\mathrm{W}$ boson helicity fractions,

$$
\omega\left(\cos \theta^{*}\right) \propto\left(1-\cos ^{2} \theta^{*}\right) f_{0}+\left(1-\cos \theta^{*}\right)^{2} f_{-}+\left(1+\cos \theta^{*}\right)^{2} f_{+}
$$

forms the basis for measurement of the $\mathrm{W}$ boson helicity.

\subsubsection{CDF Result}

The most recent analysis of the $\mathrm{W}$ boson helicity fractions measurement from CDF is performed using the Matrix Element method (ME) in which a likelihood for a sample of events is formed from a product of event probabilities calculated from the ME for a given set of event variables. Over a dataset of $2.7 \mathrm{fb}^{-1}, \mathrm{CDF}$ performed the simultaneous fit of $f_{0}$ and $f_{+}$(with the constraint $f_{-}+f_{0}+f_{+}=1$ ) to measure $f_{0}=0.88 \pm 0.11$ (stat) $\pm 0.06($ syst $), f_{+}=-0.15 \pm$ 0.07 (stat) \pm 0.06 (syst) [15].

\subsubsection{DO Result}

The D0 collaboration also made a model-independent measurement of the helicity of $\mathrm{W}$ bosons produced in top quark decays, based on a data sample of up to $2.7 \mathrm{fb}^{-1}$ of candidate $t \bar{t}$ events in the 
dilepton, and lepton + jets channels. The angle $\theta^{*}$ between the momenta of the down-type fermion and the top quark in the $\mathrm{W}$ boson rest frame for each top quark decay was measured. A fit of the resulting $\cos \theta^{*}$ distribution measures that the fraction of longitudinal $\mathrm{W}$ bosons $f_{0}=0.490 \pm$ 0.106 (stat. $) \pm 0.085$ (syst.) and the fraction of right-handed W bosons $f_{+}=0.110 \pm 0.059$ (stat. $) \pm$ 0.052 (syst.) [16].

\subsection{Forward-Backward Charge Asymmetry}

The dominant $t \bar{t}$ production mechanisms at the Tevatron $(q \bar{q} \rightarrow t \bar{t}, g g \rightarrow t \bar{t})$ do not discriminate between the final $t$ and $\bar{t}$ at Leading Order (LO), thus the differential distribution of each $t$ and $\bar{t}$ is predicted to be identical. However, the radiative corrections involving either virtual or real gluon emission lead to some differences between differential $t$ and $\bar{t}$ production, and this in turn will lead to a charge asymmetry. This charge asymmetry leads to a forward-backward angular asymmetry, $A_{F B}$, in $p \bar{p}$ collisions by CP invariance. The asymmetries originating from QCD are generally small and decrease with energy. Therefore the asymmetry measurement gives an opportunity to look for unconventional production mechanisms [17].

The D0 experiment [18] reported the first measurement of $A_{F B}$ in $p \bar{p} \rightarrow t \bar{t}+X$ production in the lepton + jets final state. This analysis used the signed difference between the rapidities of the $t$ and $\bar{t}, \Delta y=y_{t}-y_{\bar{t}}$, reflecting the asymmetry in the $t \bar{t}$, and then defined the forward-backward asymmetry to be

$$
A_{F B}=\frac{N^{\Delta y>0}-N^{\Delta y<0}}{N^{\Delta y>0}+N^{\Delta y<0}}
$$

where $N^{\Delta y>0}$ and $N^{\Delta y>0}$ are the numbers of events with positive $\Delta y$ and negative $\Delta y$, respectively. With $0.9 \mathrm{pb}^{-1}$ of data, $A_{F B}$ was found to be $0.12 \pm 0.08$ (stat.) \pm 0.01 (syst.). The leading-order SM prediction [19] is $A_{F B}=0.078 \pm 0.009$. The CDF experiment [20] also measured the same asymmetry with $1.9 \mathrm{fb}^{-1}$ of data in the $p \bar{p}$ frame and in the $t \bar{t}$ frame: $A_{F B}^{p \bar{p}}=0.17 \pm 0.08$ in the $p \bar{p}$ frame and $A_{F B}^{p \bar{p}}=0.24 \pm 0.14$ in the $t \bar{t}$ frame. These measurements are consistent with the SM prediction within $2 \sigma$.

\section{Conclusions}

Top quark properties studied at the Tevatron so far include not only mass but also charge, spin correlation, helicity, forward-backward asymmetry, width, and lifetime. The properties of the top quark at the Tevatron are consistent with the Standard Model: spin correlation and $A_{F B}$ agrees with the Standard Model within $2 \sigma$. With more data to collect, the current measurements will be improved, testing the top quark and the Standard Model more stringently. With different mechanisms of $t \bar{t}$ production than LHC, the Tevatron Top physics program will continue to play a significant role for years to come.

\section{References}

[1] Slides: http://indico.cern.ch/contributionDisplay.py?sessionId=7\& contribId=98\&confId=86184

[2] CDF Collaboration, Phys. Rev. Lett. 74, 2626 (1995) 
[3] D0 Collaboration, Phys. Rev. Lett 74, 2632 (1995)

[4] S. Willenbrock, arXiv:0807.1058v2 [hep-ph]

[5] A. F. Falk and M. E Peskin, Phys. Rev. D49, 3320 (1994)

[6] A. Quadt, Eur. Phys. J. C 48 (2006)

[7] W. Bernreuther, J. Phys. G 35083001 (2008)

[8] D. Chang, W. Chang and E. Ma, Phys. Rev. D59, 091503 (1999)

[9] CDF collaboration, CDF Conference Note 9939 (2010)

[10] D0 collaboration, Phys. Rev. Lett. 98, 041801 (2007)

[11] W. Bernreuther, A. Brandenburg, Z. G. Si and P. Uwer, Nucl. Phys. B 690, 81 (2004)

[12] The D0 collaboration, arXiv:0904.3195 [hep-ex]

[13] The D0 collaboration, D0 Conference Note 5950 (2009)

[14] The CDF collaboration, CDF Conference Note 10048 (2010)

[15] The CDF collaboration, CDF Conference Note 10004 (2009)

[16] The D0 collaboration, D0 Conference Note 5722 (2008)

[17] F. Halzen, P. Hoyer and C.S. Kim, Phys. Lett. B 195, 74 (1987)

[18] D0 Collaboration, Phys. Rev. Lett. 100, 142002 (2008)

[19] O. Antuñano, J. Kühn and G. Rodrigo, Phys. Rev. D 77, 104003 (2008)

[20] CDF Collaboration, Phys. Rev. Lett. 101, 202001 (2008) 